\title{
Methodologies and formalisms of resonance energy transfer in biophysics. Application to membrane model systems
}

\author{
Luís M. S. Loura, ${ }^{1,2}$ Rodrigo F. M. de Almeida, ${ }^{1}$ and Manuel Prieto ${ }^{1, \dagger}$ \\ ${ }^{1}$ Centro de Química-Física Molecular, Instituto Superior Técnico, P-1049-001 Lisboa, Portugal \\ 2 Departamento de Química, Universidade de Évora, Rua Romão Ramalho, 59, P-7000-671 Évora, Portugal
}

\begin{abstract}
The formalisms of resonance energy transfer (RET) to a distribution of acceptors are reviewed for several geometries relevant to membranes (planar, bilayer, multilayer) and random probe distribution. Models for nonrandom probe distribution (mean concentration model, phase separation model) are presented. Selected examples of quantitative applications of RET to these systems are described. It is illustrated how information about domain size, partition coefficients, phase composition, phase separation kinetics and bilayer aggregation can be obtained from time-resolved RET data.
\end{abstract}

\section{INTRODUCTION}

Spectroscopic techniques in general, and fluorescence spectroscopy in particular, have become indispensable tools in the study of micro-heterogeneous media. From data such as the excitation or emission spectra, fluorescence lifetimes and fluorescence anisotropy, information regarding the molecular conformation and dynamics can be obtained [1]. These techniques are helpful regarding the immediate vicinity of the studied chromophore, but do not convey information about the spatial organization features concerning the embedding medium. To this effect, there are available fluorescence techniques which rely upon distance- or concentration-dependent interaction between different species. Among them is resonance energy transfer (RET). This article reviews the formalisms of RET for different possibilities of acceptor probe distribution geometry, and illustrates them with quantitative applications to the study of model systems of biological membranes. These quasi-2D media are chosen because i) the study of biological membranes, namely the organization of multicomponent bilayers, is a very active field in biophysics and biochemistry and ii) upon varying the membrane composition (both lipid and peptide/protein components), the membrane preparation procedure and the studied chromophores, many geometrical arrangements can be materialized. Emphasis is given on the data analysis methodologies and the information that can be gained through them. Outside the scope of this review is energy migration (homotransfer), as well as RET in the rapid diffusion limit.

\section{INTRAMOLECULAR RET}

The term "intramolecular" RET is often employed to designate RET interaction between donor-acceptor

\footnotetext{
${ }^{\dagger}$ E-mail: prieto@alfa.ist.utl.pt
}

pairs for which the donor-acceptor distance is the same. This situation is likely to occur in a twochromophore molecule, provided there is spectral overlap between emission of one of them, which acts as donor, and absorption of the other, which acts as acceptor. The rate constant of energy transfer between a donor molecule, with fluorescence lifetime $\tau$, and an acceptor molecule, separated by a distance $R$, is given by [2]

$$
k_{T}=\frac{1}{\tau}\left(\frac{R_{0}}{R}\right)^{6}
$$

where $R_{0}$ is the critical distance, which can be calculated from

$$
R_{0}=0.2108 \cdot\left[k^{2} \cdot \Phi_{D} \cdot n^{-4} \cdot \int_{0}^{\infty} I(\lambda) \cdot \varepsilon(\lambda) \cdot \lambda^{4} \cdot d \lambda\right]^{16}
$$

where in turn $\kappa^{2}$ is the orientation factor (see [3] for a detailed discussion), $\Phi_{D}$ is the donor quantum yield in the absence of acceptor, $n$ is the refractive index, $\lambda$ is the wavelength, $I(\lambda)$ is the normalized donor emission spectrum, and $\varepsilon(\lambda)$ is the acceptor molar absorption spectrum. As is clear from eq. (2), $R_{0}$ can be calculated from spectroscopic data. If the $\lambda$ units used in eq. (2) are $\mathrm{nm}$, then the calculated $R_{0}$ has $\AA$ units. A quantification of the extent of RET is given by the RET efficiency, $E$, which is defined by

$$
E=1-\int_{0}^{\infty} i_{D A}(t) d t / \int_{0}^{\infty} i_{D}(t) d t
$$

In this equation, $i_{D}(t)$ and $i_{D A}(t)$ are the donor decays in absence and presence of acceptor (respectively). The effect of RET on donor fluorescence is the reduction of lifetime and quantum yield. The relationship between the donor lifetime in absence and presence of acceptor fluorophore ( $\tau_{0}$ and $\tau$, respectively) is given by

$$
E=1-\frac{\tau}{\tau_{0}}=\frac{R_{0}^{6}}{R^{6}+R_{0}^{6}}
$$




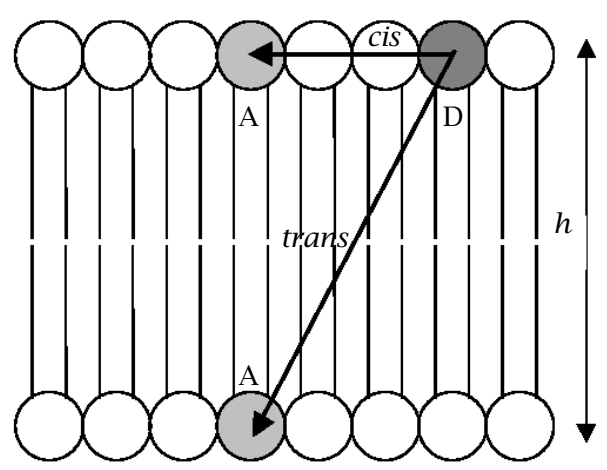

Figure 1. Schematic representation of the cis and trans RET geometries as referred to in the text. $D=$ donor probe; $A=$ acceptor probe; $h=$ distance between opposite planes of location of probes.

where $R$ is the donor-acceptor separation. An expression identical to eq. (4) can be written for the fluorescence quantum yield. From both steady-state and timeresolved data, $R$ is easily computed. This is the basis of the use of intramolecular RET as a "spectroscopic ruler" [4].

\section{RET IN INFINITE PLANAR MEDIA (RANDOM PROBE DISTRIBUTION)}

However, for many systems there is a distribution of acceptor chromophores surrounding each donor ("intermolecular" RET), and there are many relevant donoracceptor distances.

If a given donor molecule is now surrounded by $N_{A}$ acceptors (being $R_{i}$ the distance between the donor and acceptor molecule $i$ ) and $R_{0}$ is the same for every donoracceptor pair, $i_{D A}(t)$ is given by:

$$
i_{D A}(t)=\exp \left(-\frac{t}{\tau}\right) \prod_{i=1}^{N_{A}} \exp \left[\left(-\frac{t}{\tau}\right)\left(\frac{R_{0}}{R_{i}}\right)^{6}\right]
$$

In order for this equation to have practical use in data analysis, one must calculate the product on the right, which can be replaced by integration assuming a random distribution of acceptors. This procedure is also valid if there is a distribution of equivalent donors. The result for an infinite two-dimensional system (cis RET geometry, see Figure 1), assuming that there is no homotransfer between donors, the fraction of excited acceptors is negligible, translational diffusion is negligible, and the exclusion distance between donors and acceptors is much smaller than $R_{0}$, is [5]:

$$
i_{D A, c i s}(t)=\exp \left(-\frac{t}{\tau}-C\left(\frac{t}{\tau}\right)^{1 / 3}\right)
$$

where

$$
C=\Gamma\left(\frac{2}{3}\right) \cdot n \cdot \pi \cdot R_{0}^{2}
$$

In this equation, $n$ is the surface density of acceptors and $\Gamma$ is the complete gamma function. Equation (6) shows that in this situation the donor decay in presence of acceptor is no longer exponential.

Although no longer useful for distance measurement, the RET efficiency parameter is still defined by eq. (3). In this case, the integration can be carried out analytically [5], or numerically, which is preferred for multiexponentially decaying donors (and for the more complex formalisms described hereafter).

These equations should hold for RET between probes located in the same membrane leaflet of large unilamellar vesicles (LUV), because the radius of curvature $\left(\sim 100 \mathrm{~nm}\right.$, which is approximately twice the $R_{0}$ value for many useful donor-acceptor pairs) is sufficiently large for them to be treated as infinite planes in the RET scale [6].

In the past 25 years, several authors have tried to analyze RET data in model membranes using variations of the above equations. In their pioneering work, Fung and Stryer [7] studied RET between fluorescently labeled phosphatidylethanolamine (PE) in LUV of phosphatidylcholine (PC) from egg yolk. In this system, the probe distribution was expected to have bilayer (rather than planar) geometry. However, the authors considered that the opposing leaflet in the bilayer can be neglected in the calculation of $E$ if $R_{0}$ is not significantly larger than the bilayer width. Although from the variation of steady-state RET efficiency data with acceptor concentration it was possible to recover $R_{0}$ values in accordance with those obtained spectroscopically, the donor decay curves are considerably different from the theoretical ones, pointing to nonrandom probe distribution.

RET from octadecylrhodamine B (ORB) to $1,1^{\prime}, 3,3$, $3^{\prime}, 3^{\prime}$-hexamethylindotricarbocyanine $\left(\operatorname{DiIC}_{1}(7)\right)$ in fluid $16: 0,16: 0$ PC LUV [8] ( $m: n$ denotes a chain with $m$ carbon atoms and $n$ double bonds) was measured in both steady-state and time-resolved conditions. Both probes were incorporated in the outer LUV leaflet by an injection procedure, and neither of them underwent significant translocation to the inner leaflet. Hence, a planar RET geometry was expected. In this study, a modified eq. (6) was derived for biexponentially decaying donors, and the decays were globally analyzed, with linkage of donor lifetimes and pre-exponential ratio. A linear dependence of the recovered $C$ parameter as a function of acceptor concentration was verified, as expected from eq. (7). However, analysis of the decays for the same system but below the main transition temperature $\left(T_{m}\right)$ was not successful, pointing to probe aggregation in the gel phase, possibly on line defects in the gel phase structure. In this situation, the traditional framework, derived assuming random distribution of probes, is no longer valid. In section 5 we will address the problem of nonrandom probe geometry and return to this system. 


\section{RET IN INFINITE BILAYERS (RANDOM PROBE DISTRIBUTION)}

If donors and acceptors are located in infinite parallel planes (trans transfer, as often occurs in bilayers for chromophores located at different depths; see Figure 1), separated by a distance $h$, the donor decay in presence of acceptor is given by [9]:

$$
i_{D A, \text { trans }}(t)=\exp \left(-\frac{t}{\tau}-k h^{2} C F(h, t)\right)
$$

where

$$
F(h, t)=\int_{0}^{1} \frac{1-\exp \left((-1 / \tau)\left(R_{0} / h\right)^{6} \alpha^{6}\right)}{\alpha^{3}} d \alpha
$$

and $k=2 / R_{0}^{2}$.

Due to the complex time-dependence of $F$, decay analysis software is more complicated and slower for this situation, and there is the temptation to analyze data from bilayer systems with the planar formalism. It can be shown that eq. (8) reduces to eq. (6) when $h \ll R_{0}$ (as expected). However, despite for $h \approx R_{0}$ (the case for many experiments in model membranes) the RET contribution from the opposing leaflet is much smaller than RET within the same leaflet, omission of the former will lead to important quantitative deviations in the decay law. Thus, all serious quantitative analysis of RET data with bilayer probe distribution should be done with eqs. (8)-(9).

As for planar geometry systems, membrane studies for bilayer geometry have revealed accordance with the theoretical expectations for fluid bilayers, and deviations for gel bilayers. In one report, from time-resolved data of RET from $N$-(7-nitrobenz-2-oxa-1,3-diazol-4-yl) (NBD)-16:0,16:0 PE to $N$-(lissamine ${ }^{\mathrm{TM}}$-rhodamine B) (Rh)-16: 0, $16: 0$ PE in $18: 1,18: 1$ PC [10], a linear dependence of the recovered $C$ parameter as a function of acceptor concentration was verified, as expected from eq. (7), allowing the calculation of the area per lipid molecule. In another study, the time-resolved RET data for the pair NBD-16 : 0, $16: 0 \mathrm{PE} / \mathrm{Rh}-16: 0,16: 0 \mathrm{PE}$ [11] in both fluid and gel phase $16: 0,16$ : 0 PC LUV was obtained. Similarly to the pair ORB/DiIC ${ }_{1}(7)$, the analysis with eqs. (8)-(9) was successful for the fluid phase system, but not for the gel phase system, probably due to nonrandom probe distribution.

\section{EXTENSIONS TO NONRANDOM PROBE DISTRIBUTIONS}

As seen from above, even in systems for which random probe distribution was expected (one-phase lipid bilayers), deviations to the theoretical laws were observed in some cases, this being ascribed to nonuniform chromophore distribution. Several authors have tried to account for these deviations in different ways. A study of RET in Langmuir-Blodgett multilayers revealed unsatisfactory matching to the theoretical framework for random probe distribution [12]. In this example, the authors were able to globally fit the donor decays to a modified version of eq. (6):

$$
\begin{aligned}
i_{D A, \text { cis }}(t)= & (1-\alpha) \exp \left(-\frac{t}{\tau}-c t^{1 / 3}\right) \\
& +\alpha \cdot \exp \left(-\frac{t}{\tau}\right)
\end{aligned}
$$

This equation assumes that a fraction $\alpha$ of the donors might be "isolated" regarding RET (in practice, having no acceptor molecules within a distance of $2 R_{0}$ ), and show the same decay as in absence of acceptor.

An improved RET formalism that allows the possibility of nonrandom probe distribution is the mean acceptor concentration model [13]. It takes into account a continuous probability function $f(C)$ of having donors with a mean local concentration $C$ of acceptors in their surroundings, rather than a discrete function characterized by probability $\alpha$ of "seeing" no acceptors and probability $1-\alpha$ of sensing a concentration $c$, expressed by eq. (10). In this model eq. (6) (or eq. (8) for trans RET) is locally valid and the decay law is expressed as a Fredholm integral equation of the first kind regarding recovery of the function $f(C)$ :

$$
i_{D A}(t)=\int_{0}^{\infty} f(C) \exp \left(-\frac{1}{\tau}-C\left(\frac{t}{\tau}\right)^{1 / 3}\right) d C
$$

An identical equation (only the kernel $\exp (-t / \tau$ $\left.-C(t / \tau)^{1 / 3}\right)$ should be replaced by $\exp (-t / \tau-$ $\left.k h^{2} C F(h, t)\right)$ ) is valid for bilayer geometry.

This model was used to analyze the aforementioned time-resolved RET data for the pair ORB/DiIC ${ }_{1}(7)$ [14] in both fluid and gel phase $16: 0,16$ : 0 PC LUV. Narrow unimodal $f(C)$ were recovered for the fluid phase and moderate acceptor overall concentrations, confirming the random distribution of probes in this system. Higher overall acceptor concentrations (> $1 \mathrm{~mol} \%)$ resulted in wider (but still unimodal) distributions, revealing a small degree of acceptor aggregation (Figure 2E, top).

The striking results came in the analysis of gel phase data. In this case, $f(C)$ evolved from a distribution with maximum at $C=0$, for low overall acceptor concentration, to a unimodal distribution centered at higher $C$ values (see Figure 2, bottom). No evidence for donor aggregation was observed in a study of emission depolarization by homotransfer. The variation in the recovered $f(C)$ was thus ascribed to acceptor segregation in the defect lines of the gel structure. For low overall acceptor concentration, a large fraction of this probe would be located in the defects, leaving a sizeable proportion of donors as "isolated" in RET terms (peak at $C \approx 0$ ). For higher overall acceptor concentration, as more acceptor molecules are incorporated, a saturation effect probably occurs in the defects, significant amounts of acceptor incorporate in the bulk gel 

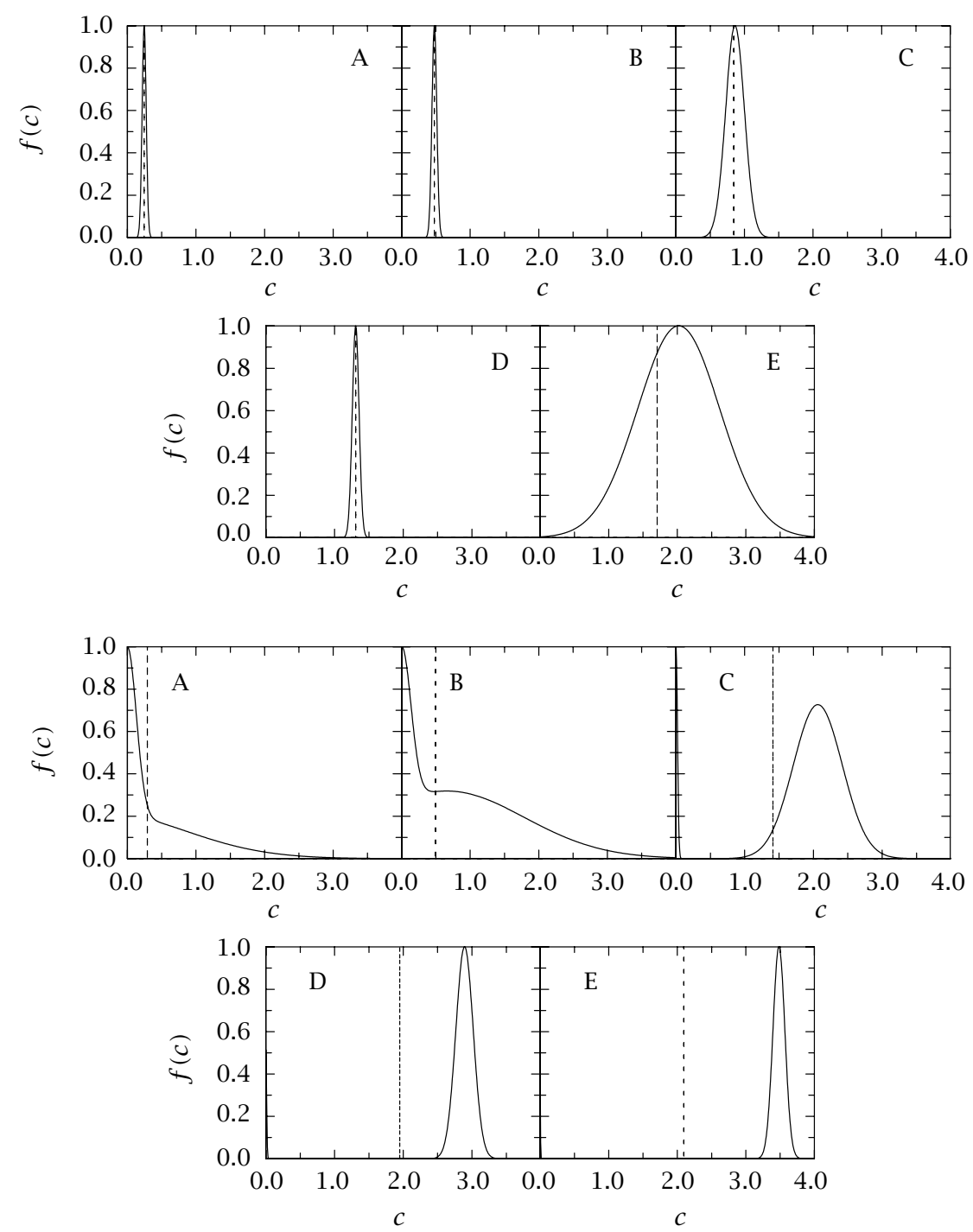

Figure 2. Solid lines: acceptor concentration distributions $f(c)$ (where $c=C \tau^{-1 / 3}$ and $C$ is defined in eq. (7)) recovered for ORB decay data in $16: 0,16: 0$ PC LUV in presence of different amounts of DiIC $C_{1}(7)$ (ratios DiIC ( $_{1}(7)$ to outer leaflet 16 : 0, 16 : 0 PC: A, 0.0022; B, 0.0044; C, 0.0082; D, 0.0126; E, 0.0171). Vertical dotted lines: $c$ values recovered for the random distribution fit (eq. (6)). Top plots: $T=50^{\circ} \mathrm{C}$ (fluid phase LUV). Bottom plots: $T=25^{\circ} \mathrm{C}$ (gel phase LUV) (Adapted from [11]).

lattice and gradually less donors remain isolated. These conclusions are supported by additional photophysical measurements [11], and agree with numerical simulations [14]. This analysis methodology was also used in the RET study of interactions between the fluorescent sterol dehydroergosterol and the polyene antibiotic filipin in small unilamellar vesicles of $16: 0,16: 0$ PC [15], revealing the formation of both filipin-sterol and filipinfilipin aggregates.

Although the above results were obtained for onephase lipid bilayers, obvious examples of nonrandom probe distribution occur in biphasic systems. In these systems, as long as the donor and acceptor probes prefer one of the two coexisting phases, RET is obviously sensitive to phase separation. For example, if the two probes show preference for the same phase, most donors will be located in a region enriched with acceptor, and it can be shown that RET efficiency increases accordingly. The opposite effect is verified if the probes partition to distinct phases. Thus, in biphasic systems, RET efficiency and probe partition are closely related. For systems with lateral phase separation (two infinite phases), the donor decay in presence of acceptor is given by [16]

$$
\begin{aligned}
i_{D A} \text {, phase separation }(t)= & A_{1} i_{D A \text {, phase } 1}(t) \\
& +A_{2} i_{D A \text {, phase } 2}(t)
\end{aligned}
$$

where $i_{D A}$ in both phases is given either by eq. (6) (for planar systems) or by eq. (8) (for bilayer systems), 


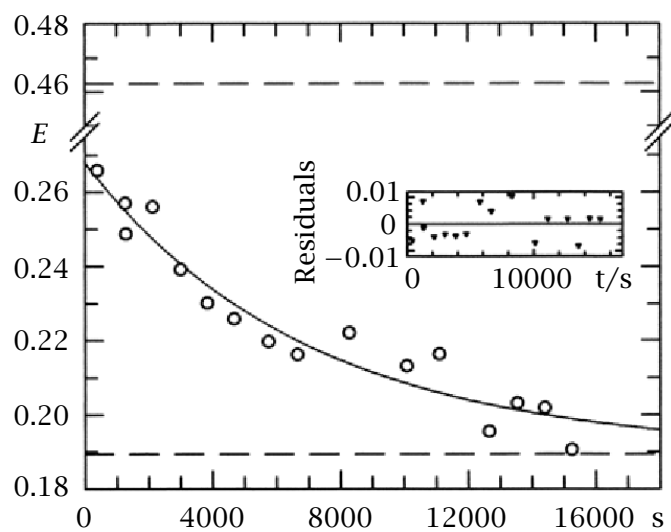

Figure 3. Energy transfer efficiency (calculated from transient-state fluorescence measurements, eq. (3)) from trans-parinaric acid to NBD-12:0,12:0 PE vs. time [20]. Experimental conditions: equimolar $12: 0,12: 0$ PC/18 : 0, 18: 0 PC LUV after a sudden thermal quench from $T_{i}=$ $65^{\circ} \mathrm{C}$ (fluid phase region) to a final temperature $T_{f}=20^{\circ} \mathrm{C}$ in the gel/fluid coexistence region. The line is the best fit of an exponential function with a non-zero limit value, and the residuals are shown in the inset. The two limiting $E$ values were calculated assuming totally random distribution of both probes at zero-time (eqs. (8)-(9); dashed line above in the plot) and complete phase separation at infinite time (eqs. (8)-(9), (12)-(13); dashed line below in the plot).

replacing $\tau$ by $\tau_{i}$, the donor excited-state lifetime in phase $i(i=1,2)$, and $C$ by $C_{i}$ which, in turn, is given by eq. (7), replacing $n$ by $n_{i}$ (the acceptor surface density in phase $i$ ), and $R_{0}$ by $R_{0 i}$, the critical RET distance for pure phase $i$. It is assumed that the probe distribution is random inside each phase (nonrandomness could be rationalized in the framework of the mean concentration model; however, that would lead to a fitting equation with a very large number of parameters for biphasic systems). The pre-exponential $A_{i}$ is proportional to the number of donor molecules in phase $i$. The decay of donor in absence of acceptor is simply

$$
i_{D}(t)=A_{1} \exp \left(-\frac{t}{\tau_{1}}\right)+A_{2} \exp \left(-\frac{t}{\tau_{2}}\right)
$$

In practice, the donor may decay biexponentially in both pure phases, and the RET geometry may not be strictly planar (cis geometry in Figure 1), because donors located in one bilayer leaflet may be able to transfer its excitation energy to acceptors located on the opposite monolayer. The necessary changes for these situations have been described [16]. In any case, the recovered parameters contain information relative to the amount of donor and acceptor in each phase. The partition coefficient of a probe between phases 1 and 2 is given by (e.g. [17]):

$$
K_{p}=\frac{P_{2} / X_{2}}{P_{1} / X_{1}}
$$

where $P_{1}$ is the probe mole fraction in lipid phase 1 , and $X_{1}$ is the lipid phase 1 mole fraction (therefore $P_{2}=1-$ $P_{1}$ and $X_{2}=1-X_{1}$ ). It is easy to show that the partition coefficients of donor $\left(K_{p D}\right)$ and acceptor $\left(K_{p A}\right)$ probes can be calculated straightforwardly from the RET decay parameters:

$$
\begin{aligned}
K_{P D} & =\frac{A_{2} / X_{2}}{A_{1} / X_{1}} \\
K_{p A} & =\frac{C_{2} \cdot a_{2} / \tau_{2}^{1 / 3}}{C_{1} \cdot a_{1} / \tau_{1}^{1 / 3}}
\end{aligned}
$$

where $a_{i}$ is the area per lipid molecule in phase $i$.

Now let $x$ represent the overall mole fraction of the lipid component which predominates in phase 2 at a given temperature, and let the phase coexistence boundaries at this temperature be $x_{1}\left(X_{2}=0\right)$ and $x_{2}\left(X_{1}=1\right)$. If at a given temperature $X_{1}$ are known for two points, $A\left(x_{A}, T\right)$ and $B\left(x_{B}, T\right)$, which are known to be located inside the phase coexistence range, $x_{1}$ and $x_{2}$, are given by [16]

$$
\begin{aligned}
& x_{1}=\frac{x_{A} \cdot X_{2 B}-x_{B} \cdot X_{2 A}}{X_{1 A}-X_{1 B}} \\
& x_{2}=\frac{x_{B} \cdot X_{1 A}-x_{A} \cdot X_{1 B}}{X_{1 A}-X_{1 B}}
\end{aligned}
$$

which allows one to calculate the compositions of phases 1 and 2 at that temperature from time-resolved RET data. If this procedure is repeated for several temperatures, the phase diagram is obtained.

These simple relationships are strictly valid only for very large domains ( $\gg R_{0}$ ). To test this formalism for a situation of phase separation into small domains, synthetic decays were generated by Monte-Carlo simulation and then globally analyzed (in presence and absence of acceptor) using eqs. (12)-(13). It was verified that even for domain size $\approx 3.5 R_{0}(\approx 15-20 \mathrm{~nm}$ for most current RET pairs), satisfactory phase boundaries estimates are recovered, whereas the $K_{p A}$ values calculated using eq. (16) are closer to unity than the input values. That is, if the acceptor prefers to incorporate in the minority phase $1\left(K_{p A}<1\right)$, but the domains of this phase are very small, donors inside those domains are still sensitive to the region outside them, and "see" a local concentration of acceptor which is smaller than the domain value $C_{1}$. The opposite happens for donors outside the domains. As a consequence, $C_{1}$ is underestimated, $C_{2}$ is overestimated, and $K_{p A}$ calculated from eq. (16) is overestimated. Conversely, if $K_{p A}>1$, but the domains of phase 1 are very small, $K_{p A}$ calculated from time-resolved RET parameters is underestimated.

Of course, $K_{p}$ values can be obtained by a plethora of established methods, including other photophysical techniques [17]. The uniqueness of RET in this respect resides in the dependence of the "apparent $K_{p}$ ", the value recovered after analysis, on the size of the phases. Other fluorescent properties often used for calculation of $K_{p}$, like fluorescence intensity, lifetime or anisotropy 
are only dependent on the immediate environment of the probe (at least for common dyes, with lifetimes smaller than $10 \mathrm{~ns}$ ), and are insensitive to the domain size. In this way, a procedure for obtaining information on the size of membrane domains would be the following:

i) Measure $K_{p}$ by distance-independent methods;

ii) Obtain time-resolved RET data and calculate $K_{p A}$ from global analysis;

iii) Compare the $K_{p A}$ values obtained in i) and ii), and from their eventual difference, conclude about domain sizes;

iv) This would allow an "educated guess", which could in turn be confirmed from adequate Monte-Carlo simulations. Theoretical decay laws would thus be obtained and compared with the experimental ones.

This discussion is valid for all biphasic systems. Experimentally, one can distinguish between gel/fluid and fluid/fluid phase separation types. In one gel/fluid heterogeneity study [18], mixtures of 12:0,12:0 PC/18:0, 18:0 PC, were investigated for two different temperatures and compositions inside the phase coexistence range. The short-tailed RET donor, NBD-12:0,12:0PE, and a short-tailed RET acceptor, 1, 1' -didodecil-3, 3, 3' , 3' -tetramethylindocarbocyanine $\left(\operatorname{DiIC}_{12}(3)\right)$, were shown to prefer the fluid phase (rich in short-tailed phospholipid) by both intrinsic anisotropy, lifetime and RET measurements, in agreement with published reports. The other studied RET acceptor, long-tailed probe $1,1^{\prime}$-dioctadecil-3, 3, 3', $3^{\prime}$ tetramethylindocarbocyanine $\left(\operatorname{DiIC}_{18}(3)\right)$, was expected to prefer the gel (rich in long-tailed phospholipid), on account of hydrophobic matching considerations [19]. While intrinsic lifetime studies indeed indicated preferential partition of $\mathrm{DiIC}_{18}(3)$ into a rigidified environment, RET analysis pointed to an increased donor-acceptor proximity as a consequence of phase separation. These apparently conflicting results were rationalized on the basis of segregation of $\mathrm{DiIC}_{18}(3)$ to the gel/fluid interphase. In order to fluid-located donors sense these interphase-located acceptors, fluid domains should be small (not exceed $\sim 10-15 \mathrm{~nm}$ ). This work shows that membrane probes which apparently prefer the gel phase may show a nonrandom distribution in this medium (in agreement with the study described above for pure DPPC gel phase LUV), and tend to locate in an environment which simultaneously leads to less strict packing constraints and to favorable hydrophobic matching interactions.

The dynamics of domain growth was also studied in the system $12: 0,12: 0 \mathrm{PC} / 18: 0,18: 0 \mathrm{PC}(1: 1$ molar ratio) [20]. LUV were first equilibrated at $65^{\circ} \mathrm{C}$, a temperature above the $T_{m}$ of both PC, where the system is in the one fluid (supposedly homogeneous) phase situation. The lipid vesicles contain also a probe that partitions preferentially to the gel (trans-parinaric acid) and another that prefers the fluid phase (NBD-12:0,12:0 PE).
Then, a sudden thermal quench to $20^{\circ} \mathrm{C}$ is carried out, and the lipid mixture is rapidly taken to the gel/fluid phase coexistence region of the phase diagram. The RET efficiency as a function of time (Figure 3) was measured and, as expected, it decreased, because as domains form and grow due to the differential partitioning of the probes, the donor is sensing a decreasing local concentration of acceptors. The process has a dynamics on the time-scale of hours. The trend of RET efficiency with time could be well described by an exponential function with a non-zero value at infinite time $(E=0.19)$. Also shown in Figure 3 is the calculated value considering infinite phase separation (eqs. (8)-(9), (12)-(13)), which is also $E=0.19$. This shows that domains are, at least 5-10 times $R_{0}$ [16].

For fluid/fluid heterogeneities, which are traditionally most difficult to characterize, these packing problems are certainly less critical. One may distinguish between fluid/fluid phospholipid heterogeneity (mixtures of two structurally different phospholipids above the $T_{m}$ of both) and liquid disordered (ld)/liquid ordered (lo) heterogeneity (e.g., PC/cholesterol mixtures). Although both types are certainly relevant as models of biomembrane heterogeneity, very few RET studies have been carried out regarding either of them. In one steady-state fluorescence work [21], the RET efficiency between the excimer of $16: 0,1$ pyrenedecanoyl PC and 4,4-difluoro-5-methyl-4-boro3a,4a-diaza-s-indacene-3-dodecanoyl,16 : 0 PC was studied as a function of composition for mixed 16 : 0,18: 1 PC/16 : 0,18: 1 phosphatidylglycerol (PG), $16: 0,18: 1 \mathrm{PC} / 16: 0,18: 1$ phosphatidylserine (PS) and $16: 0,18: 1 \mathrm{PC} / 16: 0,18: 1$ phosphatidic acid (PA) LUV, at $35^{\circ} \mathrm{C}$ (above the $T_{m}$ of all the used lipids). It was verified that $E$ increases continuously with the amount of anionic phospholipid, but, while variations in $16: 0,18: 1 \mathrm{PC} / 16: 0,18: 1 \mathrm{PG}$ LUV were small, the increase of RET efficiency was very significant in the other systems (especially in the $16: 0,18: 1 \mathrm{PC} / 16$ : 0,18 : 1 PA mixtures). These observations point to preferred colocalization of the labeled PC probes, and to probable fluid-fluid phase separation.

In the previously mentioned study of the dynamics of phase separation in $12: 0,12: 0 \mathrm{PC} / 18: 0,18$ : 0 PC [20], the expected RET efficiency for one homogeneous phase was also calculated (eqs. (8)-(9). This value ( $E=0.46$ ) was considerably higher than the value of the experimental efficiency extrapolated to time zero ( $E=0.27)$ suggesting that there was already some degree of inhomogeneity even before the phase separation process has begun.

Regarding lo/ld heterogeneity, two studies were recently carried out in the $14: 0,14: 0 \mathrm{PC} /$ cholesterol system. The phase diagram for this mixture has been determined [22]. The main focus was to probe the small domains using the strategy outlined above. In one study [23], 22-NBD-23,24-bisnor-5-colen-3 $\beta$-ol 
(NBD-cholesterol) was used as donor and ORB was used as acceptor. It was expected that NBD-cholesterol would mimic the behavior of cholesterol and partition preferably to the lo phase. However, using both steady-state fluorescence and time-resolved RET, values much less than unity were obtained for the lo/ld partition coefficients for both probes, pointing to preference for the cholesterol-poor phase. It is concluded that, in particular, NBD-cholesterol is not a suitable cholesterol analog and its distribution behavior in PC/cholesterol bilayers is in fact opposite to that of cholesterol. However, additional photophysical measurements revealed that both probes aggregate in the lo phase, preventing further characterization of the lipid domain structure.

In the other study [16], NBD-14:0,14:0 PE and Rh-14 : 0,14:0 PE were used as donor and acceptor, respectively. Although Rh-14:0,14:0 PE prefers the ld (or $\alpha$ ) phase, NBD-14: 0,14:0 PE prefers the lo (or $\beta$ ) phase, as determined by fluorescence intensity and anisotropy variations, respectively. Accordingly, RET efficiency decreases as a consequence of phase separation. For $x_{\text {chol }}=0.15$ and $x_{\text {chol }}=0.20$ (the studied samples with smaller $X_{\beta}$ in the lo/ld coexistence range), the experimental $C_{\alpha}$ value (which would always be expected to be larger than $C_{\beta}$ ) is smaller than expected, while the opposite is true for $C_{\beta}$. This, together with numerical simulations of decays in biphasic systems, suggests that in this region of the phase diagram, the lo domains, dispersed in ld phase, should be very small (of the order of magnitude of $R_{0}$, that is, a few $\mathrm{nm}$ ). On the other hand, domains of ld in the cholesterol-rich end of the coexistence range have comparatively large size. When the phase boundaries are calculated using eqs. (17)-(18), very good agreement with the literature is obtained in the cholesterol-rich end (both 0.28 at $30^{\circ} \mathrm{C}$ ), while a considerable larger value is obtained in the cholesterol-poor end $(0.18$ from RET, compared to $\approx 0.075$ from [22]). These results do not necessarily contradict the published diagram, which was also confirmed by domain size-independent fluorescence measurements [24]. They stem from the distance dependence of RET and the existence of small domains. In other words, there may be phase separation below $x_{\alpha}<0.15$, but, if this is the case, the domains should be very small. Ideally, the published and RET coexistence boundaries should coincide if the phases are large, as observed at the other end of the tie-line.

\section{RET IN INFINITE MULTILAYERS (RANDOM PROBE DISTRIBUTION)}

Monolayers and bilayers are not the end of the story regarding the possibilities of RET geometry in model membranes. We shall illustrate this with a brief description of our study of the interaction of the polylysine peptide $\mathrm{K}_{6} \mathrm{~W}$ with equimolar 16 : 0, 16 : 0 PC/16 : 0, 16 : 0 PS LUVs.
Because of the high positive charge of the peptide, reorganization of the zwitterionic lipid (PC)/acidic lipid (PS) mixture was expected. This could be detected by the variation of RET efficiency between fluorescently labeled PC analogs upon incorporation of peptide. To this extent, 2-[3-(diphenylhexatrienyl)propanoyl]1-hexadecanoyl-sn-glycero-3-phosphocholine (DPH-PC) was used as donor and 1-palmitoyl-2-[12-(7-nitrobenz-2 - oxa-1, 3 - diazol - 4-yl)aminododecanoyl] - sn-glycero3-phosphocholine (NBD-PC) was used as acceptor $\left(R_{0}\left(45^{\circ} \mathrm{C}\right)=41 \AA ; R_{0}\left(60^{\circ} \mathrm{C}\right)=40 \AA\right)$. Two distinct situations were considered: at $T=60{ }^{\circ} \mathrm{C}$, above the $T_{m}$ of both phospholipids, there is a single fluid lipid phase; at $T=45^{\circ} \mathrm{C}$, between the $T_{m}$ values for $16: 0,16: 0 \mathrm{PC}$ and $16: 0,16: 0 \mathrm{PS}$, there is gel/fluid phase coexistence. We sought to determine whether the insertion of this basic peptide would lead to: $(i)$ phase separation from a single fluid phase $16: 0,16: 0 \mathrm{PC} / 16: 0,16$ : 0 PS mixture $\left(T=60^{\circ} \mathrm{C}\right)$ and, (ii) noticeable reorganization of pre-existing gel/fluid domains $\left(T=45^{\circ} \mathrm{C}\right)$. The former effect would be clearly apparent in a RET measurement, as segregation of a $16: 0,16: 0$ PC-rich phase (containing most of the probe molecules) would lead to local enrichment of acceptor probe, and thus higher RET efficiency. The latter effect would be visible for example if the pre-existing domains were very small $(\sim 10 \mathrm{~nm}$ or smaller), and became considerably larger upon incorporation of peptide.

As a first approach, fluorescence intensity decays were described by empirical sum-of-exponential functions and the recovered fitting functions were integrated to calculate the FRET efficiency $E$ (eq. (3)). The value obtained for this parameter at $60{ }^{\circ} \mathrm{C}$ in the absence of peptide is approximately 0.40 , close to the theoretical expectation for a random distribution of acceptors (0.43), which can be computed from eqs. (3), (8) and (9). In this calculation, $h=1.7 \mathrm{~nm}$ (corresponding to half the bilayer width [25]) was considered, because, whereas it is a good approximation to consider the DPH moiety of DPH-PC located in the center of the bilayer, the NBD group of NBD-PC snorkels to the bilayer interface [26] (see Figure 4, left). When peptide is added, $E$ increases up to 0.54 (for a peptide:lipid ratio of $1: 13$ ), seeming to indicate phase separation. On the other hand, addition of peptide to the gel/fluid mixture $\left(45^{\circ} \mathrm{C}\right)$ does not lead to a significant increase in $E$, i.e., there was no detectable rearrangement of the preexisting lipid domains.

Despite being a rapid way of obtaining a first glimpse into the possible membrane organization, the steady-state analysis information is always limited. The FRET efficiency parameter results from time integration of the actual decay law, and in this process considerable detail regarding the probes distribution is lost. Thus, whenever possible, a complete parameter analysis of the time-resolved fluorescence donor decays should be carried out. In this experiment, analysis of the decays 


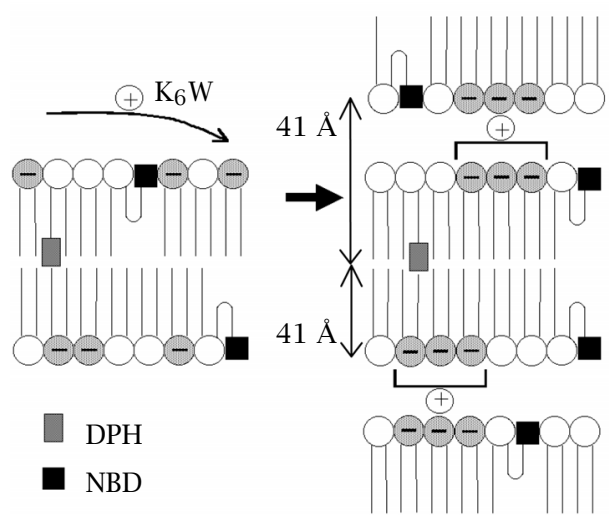

Figure 4. Schematic representation of the formation of multibilayers from $16: 0,16: 0$ PC/16:0,16:0 PS fluid $L U V$ in the presence of $K_{6} W$, as revealed from the timeresolved fluorescence data analysis (see text for details).

recorded at $45^{\circ} \mathrm{C}$ was made using eq. (12) (together with eqs. (8) and (9)), and from the essentially invariant recovered parameters, it was possible to conclude that addition of peptide does not indeed lead to appreciable changes in domain organization. On the other hand, it was not possible to analyze the decays recorded at $60{ }^{\circ} \mathrm{C}$ in the presence of peptide with the same model. For these samples, a different regime was observed in the decay profile: at longer times, the decay in presence of acceptor did not become parallel (in a log scale) to that without acceptor, as it should according to eqs. (8)(9). Successful analysis required the use of a modification of eq. (8), allowing for FRET to a second layer of acceptors:

$$
\begin{aligned}
& i_{D A, \operatorname{trans}}(t) \\
& \quad=\exp \left(-\frac{t}{\tau}-k C\left(h_{1}^{2} F\left(h_{1}, t\right)+h_{2}^{2} F\left(h_{2}, t\right)\right)\right)
\end{aligned}
$$

In this equation, $h_{1}$ and $h_{2}$ are now the distances between the plane of the donors and the two distinct planes of acceptors. When $h_{1}=1.7 \mathrm{~nm}$ was assumed (see justification above), the most successful analysis was obtained for $h_{2}=4.1 \mathrm{~nm}$. No evidence for phase separation was apparent from the decay analyses.

A possible explanation for these results is that the value recovered for $h_{2}$ corresponds to RET to acceptors located in a different lipid bilayer to that of the donors. In this scenario, $\mathrm{K}_{6} \mathrm{~W}$ promotes bilayer aggregation of $16: 0,16: 0 \mathrm{PC} / 16: 0,16: 0$ PS bilayers LUV in the fluid phase, possibly leading to formation of small anionic lipid clusters within each bilayer, but without leading to detectable phase separation (Figure 4, right). This finding agrees with a microscopy study [27] who also ruled out the induction of large domains by basic peptides in mixtures containing acidic lipids. Of course, in the framework of our model, there would be at least a third layer of acceptors located at approximately $h_{3}=(h 2+$ one bilayer width $) \cong 7.5 \mathrm{~nm}$.
However, the contribution of RET to this plane (and further planes) of acceptors would be much smaller and effectively masked by RET to acceptors located at $h_{1}$ and $h_{2}$.

Other systems for which multilayer geometry is expected are cationic lipid/DNA complexes (lipoplexes), for which a "sandwich" arrangement, with alternate DNA and lipid lamellar layers has been proposed [28]. These aggregates have aroused great interest in the biochemical community because of the possibility of their use in gene therapy [29]. Studies of characterization of lipoplexes using the described RET methodologies are currently underway in our laboratory.

\section{ACKNOWLEDGMENTS}

This work was funded by Program POCTI, FCT, Portugal. R. F. M. de A. acknowledges a grant from POCTI (BD 943/2000).

\section{REFERENCES}

[1] J. R. Lakowicz, Principles of Fluorescence Spectroscopy, 2nd ed., Plenum Press, New York, 1999.

[2] Th. Z. Förster, Naturforsch. 4a (1949), 321.

[3] B. Van Der Meer, G. Coker III, and S.-Y. S. Chen, Resonance Energy Transfer: Theory and Data, VCH Publishers, New York, 1994.

[4] L. Stryer, Ann. Rev. Biochem. 47 (1978), 829.

[5] P. K. Wolber and B. S. Hudson, Biophys. J. 28 (1979), 197.

[6] J. Eisinger, W. E. Blumberg, and R. E. Dale, Ann. N. Y. Acad. Sci. 366 (1981), 155.

[7] B. K. Fung and L. Stryer, Biochemistry 17 (1978), 5241.

[8] L. M. S. Loura, A. Fedorov, and M. Prieto, Biophys. J. 71 (1996), 1823.

[9] L. Davenport, R. E. Dale, R. H. Bisby, and R. B. Cundall, Biochemistry 24 (1985), 4097.

[10] G. Lantzsch, H. Binder, and H. Heerklotz, J. Fluorescence 4 (1994), 339.

[11] L. M. S. Loura, A. Fedorov, and M. Prieto, J. Phys. Chem. B. 104 (2000), 6920.

[12] P. M. Ballet, M. Van der Auweraer, F. C. De Schryver, H. Lemmetyinen, and E. Vourimaa, J. Phys. Chem. 100 (1996), 13701.

[13] Y. S. Liu, L. Li, S. Ni, and M. Winnik, Chem. Phys. 177 (1993), 579.

[14] L. M. S. Loura and M. Prieto, J. Phys. Chem. B 104 (2000), 6911.

[15] L. M. S. Loura, M. A. R. B. Castanho, A. Fedorov, and M. Prieto, Biochim. Biophys. Acta 1510 (2001), 125.

[16] L. M. S. Loura, A. Fedorov, and M. Prieto, Biophys. J. 80 (2001), 776.

[17] L. Davenport, Meth. Enzymol. 278 (1997), 487.

[18] L. M. S. Loura, A. Fedorov, and M. Prieto, Biochim. Biophys. Acta 1467 (2000), 101. 
[19] O. G. Mouritsen and M. Bloom, Biophys. J. 46 (1984), 141.

[20] R. F. M. de Almeida, L. M. S. Loura, A. Fedorov, and M. Prieto, Biophys. J. 82 (2002), 823.

[21] T. Ahn and C.-H. Yun, J. Biochem (Tokyo) 124 (1998), 622.

[22] P. F. F. Almeida, W. L. C. Vaz, and T. E. Thompson, Biochemistry 31 (1992), 6739.

[23] L. M. S. Loura, A. Fedorov, and M. Prieto, Biochim. Biophys. Acta 1511 (2001), 236.

[24] C. R. Mateo, A. U. Acuña, and J.-C. Brochon, Biophys. J. 68 (1995), 978.
[25] D. Marsh, Handbook of Lipid Bilayers, CRC Press, Boca Raton, FL, 1990.

[26] A. Chattopadhyay and E. London, Biochemistry 26 (1987), 39.

[27] D. Murray, A. Arbuzova, G. Hangyas-Mihalyne, A. Gambhir, N. Ben-Tal, B. Honig, and S. McLaughlin, Biophys. J. 77 (1999), 3176.

[28] J. O. Rädler, I. Koltover, T. Salditt, and C. R. Safinya, Science 275 (1997), 810.

[29] L. Huang, M.-C. Hung, and E. Wagner, Non Viral Vectors for Gene Therapy, Academic Press, San Diego, CA, 1999. 


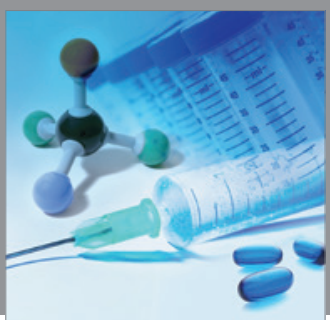

International Journal of

Medicinal Chemistry

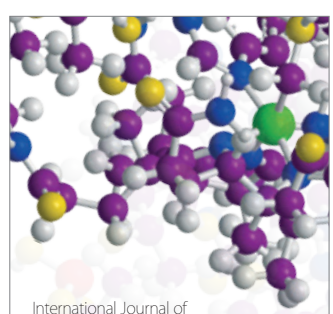

Carbohydrate Chemistry

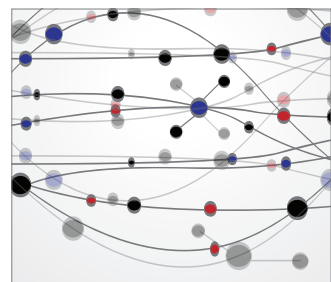

The Scientific World Journal
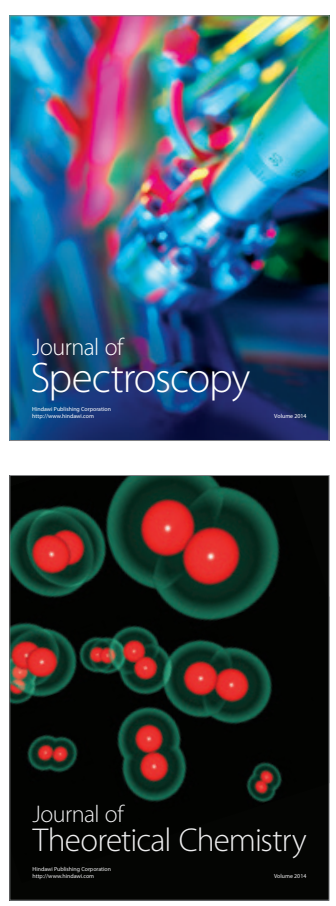
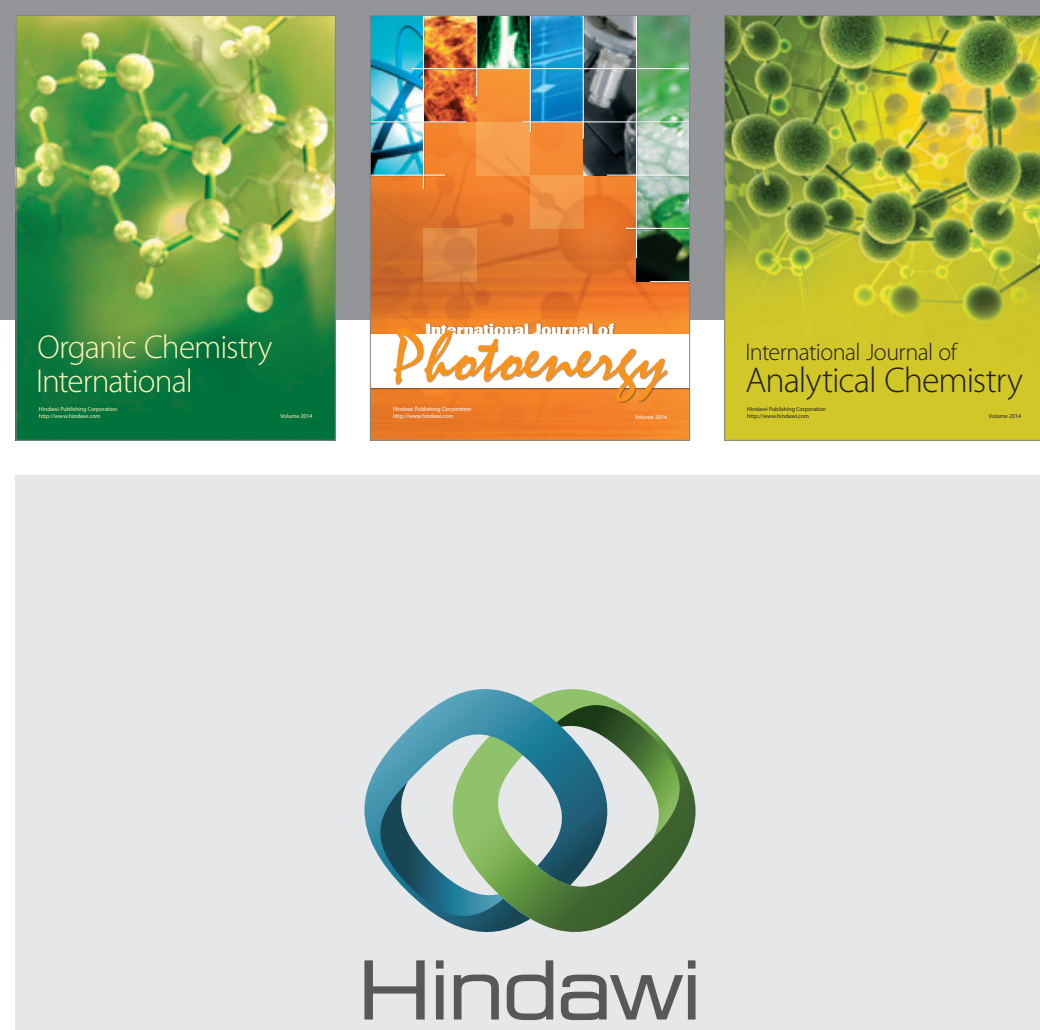

Submit your manuscripts at

http://www.hindawi.com
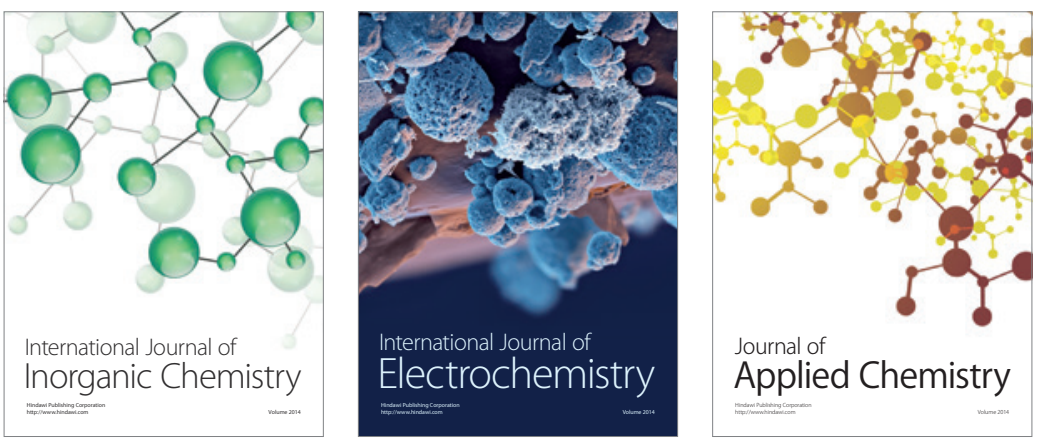

Journal of

Applied Chemistry
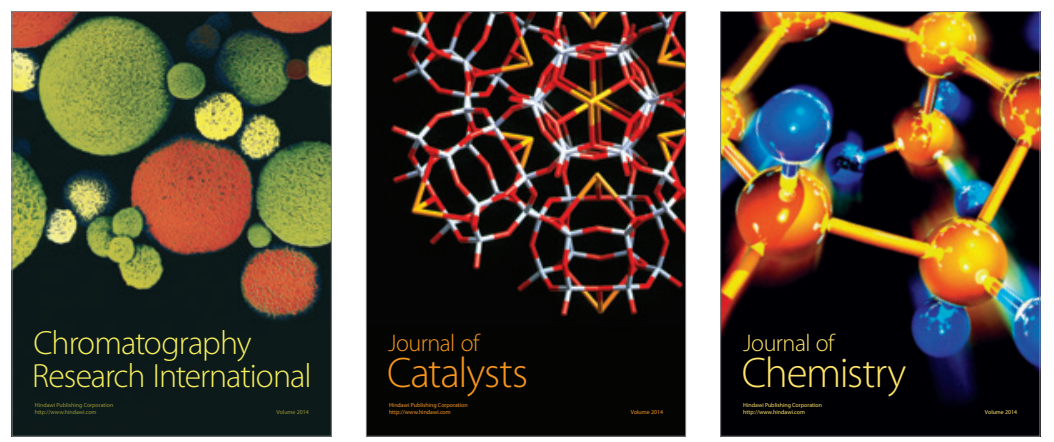
